\title{
ESTIMATING KNOWLEDGE DECAY
}

\author{
John Debenham \\ University of Technology, Sydney
}

\begin{abstract}
Knowledge 'decay' is a measure of the degradation of knowledge integrity. In a unified knowledge representation, data, information and knowledge are all represented in a single formalism as "items". A unified knowledge representation is extended here to include two measures of knowledge integrity. A rule of inference is defined on the unified knowledge representation that preserves the validity of these two measures. This rule of inference is used to define a normalised knowledge base. The use of a unified knowledge representation and the application of knowledge base normalisation simplify the estimation of knowledge decay.
\end{abstract}

Keywords integrity management, knowledge-base design

\section{INTRODUCTION}

Knowledge 'decay' is a measure of the degradation of knowledge integrity. In a unified knowledge representation, data, information and knowledge are all represented in a single formalism as "items". A unified model is expressed in terms of "items" and "objects" [1]; objects are itembuilding operators. A rule of inference, called item (and object) "join" is defined [2]. That rule is extended here so that it accommodates the two measures of knowledge integrity. A single rule for" knowledge decomposition" is defined in terms of that rule. Knowledge decomposition is applied to normalise the knowledge in a knowledge base. Classical database normalisation [3] is a special case of knowledge base normalisation. The use of a unifiedknowledge representation and the application of knowledge base normalisation simplifies the estimation of knowledge decay.

The original version of this chapter was revised: The copyright line was incorrect. This has been corrected. The Erratum to this chapter is available at DOI: 10.1007/978-0-387-35602-0_35 


\section{UNIFIED KNOWLEDGE REPRESENTATION}

The terms 'data', 'information' and 'knowledge' are used here in a rather idiosyncratic sense [1]. The data in an application are those things that can be represented as simple constants or variables. The information is those things that can be represented as tuples or relations. The knowledge is those things that can be represented either as programs in an imperative language or as rules in a declarative language. Items are a unified knowledge representation; they have a uniform format no matter whether they represent 'data', 'information' or 'knowledge' things.

The key to the unified representation is the way that the meaning of an item, the item 'semantics' is specified. The semantics of an item is a function that recognises the members of the "value set" of that item. The value set of an information item is the set of tuples that are associated with a relational implementation of that item. Knowledge items, including complex, recursive knowledge items, have value sets too [1]. For example, the item, which represents the rule "the sale price of parts is the cost price marked up by a universal mark-up factor", could have a value set of quintuples associated with [part/sale-price, part/cost-price, mark-up].

The notion of an item is extended here to incorporate two integrity measures. The item tuple integrity measure for an item is a measure of the likelihood that a given tuple should not belong to the value set of that item. The item set integrity measure for an item is a measure of the likelihood that a given set is an invalid value set of that item. Items are either represented informally as " $\mathrm{i}$-schema" or formally as $\lambda$-calculus expressions. The $\mathrm{i}$-schema notation is used in applications. Formally, given a unique name $A$, an n-tuple $\left(m_{1}, m_{2}, \ldots, m_{n}\right), M=\Sigma_{i} m_{i}$, if:

- $S_{A}$ is an $M$-argument expression of the form:

$$
\lambda \mathrm{y}_{1}^{1} \ldots \mathrm{y}_{m_{1}}^{1} \ldots \mathrm{y}_{m_{n}}^{n} \bullet\left[S_{A_{1}}\left(\mathrm{y}_{1}^{1}, \ldots, \mathrm{y}_{m_{1}}^{1}\right) \wedge . . \wedge S_{A_{n}}\left(\mathrm{y}_{1}^{n}, \ldots, \mathrm{y}_{m_{n}}^{n}\right) \wedge J\left(\mathrm{y}_{1}^{1}, \ldots, \mathrm{y}_{m_{1}}^{1}, \ldots, \mathrm{y}_{m_{n}}^{n}\right)\right] \bullet
$$

where $\left\{A_{l}, \ldots, A_{n}\right\}$ is an ordered set of not necessarily distinct items, each item in this set is called a component of item $A$.

- $\mathrm{V}_{A}$ is an $\mathrm{M}$-argument fuzzy expression of the form:

$$
\lambda \mathrm{y}_{1}^{1} \ldots \mathrm{y}_{m_{1}}^{1} \ldots \mathrm{y}_{m_{n}}^{n} \bullet\left[V_{A_{1}}\left(\mathrm{y}_{1}^{1}, \ldots, \mathrm{y}_{m_{1}}^{1}\right) \wedge . . \wedge V_{A_{n}}\left(\mathrm{y}_{1}^{n}, \ldots, \mathrm{y}_{m_{n}}^{n}\right) \wedge K\left(\mathrm{y}_{1}^{1}, \ldots, \mathrm{y}_{m_{1}}^{1}, \ldots, \mathrm{y}_{m_{n}}^{n}\right)\right] \bullet
$$
where $\left\{A_{1}, \ldots, A_{n}\right\}$ are the components of item $A, \mathrm{~K}$ is a fuzzy predicate and $1 / 4$ is the fuzzy "min" conjunction.

- $\mathrm{C}_{A}$ is a fuzzy expression of the form:

$$
\left[\mathrm{C}_{\mathrm{A}_{1}} \wedge \mathrm{C}_{\mathrm{A}_{2}} \wedge \ldots \wedge \mathrm{C}_{\mathrm{A}_{\mathrm{n}}} \wedge(L)_{\mathrm{A}}\right]
$$

where $\wedge$ is the fuzzy "min" conjunction and $L$ is a fuzzy expression constructed as a logical combination of: 
- $\operatorname{Card}_{A}$ lies in some numerical range;

- Uni $\left(A_{i}\right)$ for some $\mathrm{i}, 1 \leq \mathrm{i} \leq \mathrm{n}$, and

- $\operatorname{Can}\left(A_{i}, \mathrm{X}\right)$ for some $\mathrm{i}, 1 \leq \mathrm{i} \leq \mathrm{n}$, where $\mathrm{X}$ is a non-empty subset of $\left\{A_{1}, \ldots, A_{n}\right\}-\left\{A_{i}\right\}$;

subscripted with the name of the item $A$,

then the named triple $A\left[\mathrm{~S}_{A}, \mathrm{~V}_{A}, \mathrm{C}_{A}\right]$ is an M-adic item with item name $A, \mathrm{~S}_{A}$ is called the item semantics of $A, \mathrm{~V}_{A}$ is called the item tuple integrity measure of $A$ and $\mathrm{C}_{A}$ is called the item set integrity measure of $A$. "Uni( $\left.A_{i}\right)$ " is a fuzzy predicate whose truth value is "the proportion of the members of the value set of item $A_{i}$ that also occur in the value set of item $A$ ". "Can $\left(A_{i}, \mathrm{X}\right)$ " is a fuzzy predicate whose truth value is "in the value set of item $A$, the proportion of members of the value set of the set of items $X$ that functionally determine members of the value set of item $A_{i}$,". "Card ${ }_{A}$ " means "the number of different values in the value set of item $A$ ". The subscripts identify the item's components to which that measure applies. Given an item $A$ and tuple $\mathrm{X}$, if $\mathrm{V}_{A}(\mathrm{X})=1$ then this does not imply that $\mathrm{X}$ is invalid in the value set of $A$; if $\mathrm{V}_{A}(\mathrm{X})=0$ then $\mathrm{X}$ is invalid in the value set of $A$. Likewise for $\mathrm{C}_{A}$. The measures are not measures of validity. A value of unity does not imply validity. An item's semantics specifies what should be in an implementation, and the two integrity measures are measures of invalidity of what is in an implementation.

For example, an application may contain an association whereby each cost-price is associated with a sales-tax amount as determined by the prevailing tax-rate. This association could be represented by the knowledge item:

[cost-price, sales-tax, tax-rate][

$$
\begin{aligned}
& \lambda \mathrm{xyz}^{\bullet}\left[\mathrm{S}_{\text {cost-price }}(\mathrm{x}) \wedge \mathrm{S}_{\text {sales-tax }}(\mathrm{y}) \wedge \mathrm{S}_{\text {tax-rate }}(\mathrm{z}) \wedge(\mathrm{y}=\mathrm{x} \times \mathrm{z})\right] \bullet \\
& \lambda \mathrm{xyz} \bullet\left[\mathrm{V}_{\text {cost-price }}(\mathrm{x}) \wedge \mathrm{V}_{\text {sales-tax }}(\mathrm{y}) \wedge \mathrm{V}_{\text {tax-rate }}(\mathrm{z}) \wedge \mathrm{K}_{2}(\mathrm{x}, \mathrm{y}, \mathrm{z})\right] \bullet \\
& {\left[\mathrm{C}_{\text {cost-price }} \wedge \mathrm{C}_{\text {sales-tax }} \wedge \mathrm{C}_{\text {tax-rate }} \wedge\right.} \\
& \quad(\text { Can }(\text { cost-price, }\{\text { sales-tax, tax-rate }\}) \wedge \\
& \quad \text { Can }(\text { sales-tax, }\{\text { cost-price,tax-rate }\}) \wedge \\
& \left.\left.\quad \text { Can }(\text { tax-rate, }\{\text { cost-price, sales-tax }\}))_{\text {[cost-price, sales-tax, tax-rate }]}\right]\right]
\end{aligned}
$$

where: 


$$
K_{2}(x, y, z)= \begin{cases}0 & \text { if } x \neq 0 \text { and } y \neq 0 \\ \frac{x}{x+100 \times|z \times x-y|} & \text { otherwise }\end{cases}
$$

Items make it difficult to analyse the structure of the whole application because, for example, two rules that share the same basic wisdom may be expressed in terms of quite different components; this could obscure their common wisdom. To make the inherent structure of knowledge clear 'objects' are introduced as item building operators [4].

Object names are written in bold italics. Suppose that the conceptual model already contains the item "part" which represents spare parts, and the item "cost-price" which represents cost prices; then the information "spare parts have a cost price" can be represented by "part/cost-price" which may be built by applying the "costs" object to part and cost-price:

part/cost-price $=$ costs (part, cost-price)

Suppose that the conceptual model already contains the item "part/sale-price" which represents the association between spare parts and their corresponding selling price, and the item "mark-up" which represents the data thing a universal mark-up factor; then the rule "spare parts are marked up by a universal mark up factor" can be represented by [part/sale-price, part/cost-price, mark-up] which is built by applying the "mark-up-rule" object to the items "part/sale-price", "part/cost-price" and "mark-up":

[part/sale-price, part/cost-price, mark-up] $=$ mark-up-rule(part/sale-price, part/cost-price, mark-up)

The conceptual model contains items. A fundamental set of data items in the conceptual model is called the basis. The remaining items in the conceptual model are built by applying object operators to the other items in the conceptual model. As for items, objects may either be represented informally as "o-schema" or formally as typed $\lambda$-calculus expressions.

In [2] the composition of items and objects using a "join" operator is described. Join is extended here to include the measures of integrity. Decomposition is defined in terms of join. Knowledge base normalisation is defined in terms of decomposition [5]. Normalisation removes hidden relationships from the conceptual model. Hidden relationships can present a maintenance hazard [6]. Knowledge base normalisation simplifies the estimation of knowledge decay [7].

Item join provides the basis for item decomposition. The definition of "item composition" follows. Given two items: 
$A\left[\mathrm{~S}_{A}, \mathrm{~V}_{A}, \mathrm{C}_{A}\right]$ and

$B\left[\mathrm{~S}_{B}, \mathrm{~V}_{B}, \mathrm{C}_{B}\right]$

Suppose that $\mathrm{S}_{A}$ has $\mathrm{n}$ variables, that is $A$ is an $\mathrm{n}$-adic item. Suppose that $\mathrm{S}_{B}$ has $\mathrm{m}$ variables, that is $B$ is an m-adic item. Some of the components of $A$ and $B$ may be identical. Suppose that k pairs of components of $A$ and $B$ that are identical are identified, where $k \geq 0$. Let $E$ be an ordered set of components where each is one of these identical pairs of components of both $A$ and $B$. E may be empty. To ensure that the definition is well defined the order of the components in the set $\mathrm{E}$ is the same as order in which they occur as components of $A$. Suppose the semantics expressions of the components from item $A$ (or item $B$ ) that are in the set $\mathrm{E}$ are expressed in terms of a total of $\mathrm{p}$ variables. Let $A^{*}$ be an $\mathrm{n}$-adic item that is identical to item $A$ except for the order of its variables. The last $\mathrm{p}$ variables in $A^{*}$ are those variables in $A$ that belong to the components of $A$ in the set $\mathrm{E}$. Let $B^{*}$ be an m-adic item that is identical to item $B$ except for the order of its variables. The first $\mathrm{p}$ variables in $B^{*}$ are those variables in $B$ that belong to the components of $B$ in the set $\mathrm{E}$. Let $\pi$ be a permutation that turns the ordered set of variables of $A^{*}$ into the ordered set of variables of $A$. Let $\pi$ ' be a permutation that turns the ordered set of variables of $B *$ into the ordered set of variables of $B$. Suppose that ' $\mathrm{x}$ ' is an (n-p)-tuple of free variables, ' $y$ 'is a p-tuple of free variables and ' $z$ ' is an (m-p)-tuple of free variables. Then the item with name $A \otimes_{\mathrm{E}} B$ is the composition of $A$ and $B$ on $\mathrm{E}$ and is defined to be the item:

$\left(A \otimes_{\mathrm{E}} B\right)\left[\lambda \mathrm{xyz} \cdot\left[\mathrm{S}_{A}(\pi(\mathrm{x}, \mathrm{y})) \wedge \mathrm{S}_{B}\left(\pi^{\prime}(\mathrm{y}, \mathrm{z})\right)\right]\right.$

$$
\left.\lambda \mathrm{xyz} \cdot\left[\mathrm{V}_{A}(\pi(\mathrm{x}, \mathrm{y})) \wedge \mathrm{V}_{B}\left(\pi^{\prime}(\mathrm{y}, \mathrm{z})\right)\right] \cdot, \mathrm{C}_{A \otimes_{\mathrm{E}} B}\right]
$$

where $\mathrm{C}_{A} \otimes_{\mathrm{E}} B$ is defined as follows. Suppose that $\mathrm{C}_{A}$ is an expression of the form $c_{A} \wedge \mathrm{G}$ where $\mathrm{c}$ is that part of $\mathrm{C}_{A}$ that carries the subscript ' $A$ ' where any occurrence of the predicate Card are equated to "true", and $\mathrm{G}$ is that part of $C_{A}$ that carries subscripts other than ' $A$ '. Likewise suppose that $C_{B}$ is an expression of the form $\mathrm{d}_{B} \wedge \mathrm{H}$. Then:

$\mathrm{C}_{A \otimes_{\mathrm{E}} B}=(\mathrm{c} \wedge \mathrm{d})_{A \otimes_{\mathrm{E}} B^{\wedge}}(\mathrm{G} \wedge \mathrm{H})$

The set $\mathrm{E}$ is $a$ set of identical pairs of components of $A$ and $B$. If $\mathrm{E}$ is the set of all identical pairs of components of $A$ and $B$ then $A \otimes_{\mathrm{E}} B$ is written as $A \otimes B$.

Given two n-adic items $A$ and $B$ with the same set of components that are not necessarily in the same order, $A$ and $B$ are weakly equivalent, written $A \cong_{\mathrm{w}} B$, if there exists a permutation $\pi$ such that:

$\left(\forall \mathrm{x}_{1} \mathrm{x}_{2} \ldots \mathrm{x}_{\mathrm{n}}\right)\left[\mathrm{S}_{A}\left(\mathrm{x}_{1}, \mathrm{x}_{2}, \ldots, \mathrm{x}_{\mathrm{n}}\right) \leftrightarrow \mathrm{S}_{B}\left(\pi\left(\mathrm{x}_{1}, \mathrm{x}_{2}, \ldots, \mathrm{x}_{\mathrm{n}}\right)\right)\right]$ 
where the $\mathrm{x}_{\mathrm{i}}$ are the $\mathrm{n}_{\mathrm{i}}$ variables associated with the $\mathrm{i}$ th component of $A$. The composition $A \otimes_{\mathrm{E}} B$ is a monotonic composition if $A \otimes_{\mathrm{E}} B$ is not weakly equivalent with either $A$ or $B$.

The $\otimes$ operator forms the basis of decomposition in which each item may be replaced by a set of simpler items. An item $I$ is decomposable into the set of items $D=\left\{I_{1}, I_{2}, \ldots, I_{n}\right\}$ if: $I_{i}$ has non-trivial semantics for all $\mathrm{i}$, $\mathrm{I}=I_{I} \otimes I_{2} \otimes \ldots \otimes I_{n}$, where each composition is monotonic. If item $I$ is decomposable then it will not necessarily have a unique decomposition [4]. A knowledge base is normal if it contains no decomposable items [1].

\section{KNOWLEDGE BASE IMPLEMENTATION}

The semantics of an item $A\left[\mathrm{~S}_{A}, \mathrm{~V}_{A}, \mathrm{C}_{A}\right]$ is a function that recognises the members of its value set. The value set is a conceptual notion in the system design. So the value set of the item $A$-as in the definition of an $\mathrm{M}$-adic item above-at time $\tau$ is:

$$
\gamma^{\tau}(A)=\left\{\mathrm{y}_{1}^{1} \ldots \mathrm{y}_{m_{1}}^{1} \ldots \mathrm{y}_{m_{n}}^{n}: S_{A}\left(\mathrm{y}_{1}^{1}, \ldots, \mathrm{y}_{m_{1}}^{1}, \ldots, \mathrm{y}_{m_{n}}^{n}\right) \text { at time } \tau\right\}
$$

A knowledge base implementation is a set of knowledge items and a set of stored relations and data domains representing some information and data items. Some information and data items are associated with actual stored data, and some are not. Knowledge items are not normally associated with actual stored data. If an item is associated with actual stored data then it is a real item; otherwise it is a virtual item. The set of tuples in the implementation of the real item $A$ is denoted by $\lambda^{\alpha}(A)$ where $\alpha$ is the time of the most recent modification to those tuples. Knowledge items may be used to derive tuples for virtual data and information items. For example, suppose that the real data item mark-up has a stored data value mark-up, and that the real information item part/cost-price has a stored relation part/cost-price. Then the knowledge item [part/sale-price, part/cost-price, mark-up]-or an "if-then" implementation of it-may be used to derive tuples in the relation for the virtual item part/sale-price. Further, the knowledge item [part/sale-price, part/tax-payable, tax-rate] could then enable the tuples in the relation for the virtual item part/tax-payable to be derived. This is illustrated in Fig. 1. If a virtual item $A_{i}$ is a component of a knowledge item $A$ where the tuples (or data values) associated with $A_{i}$ are derived from $\left\{\lambda^{\alpha_{\mathrm{j}}}\left(A_{j}\right): A_{j}\right.$ is a component of $\left.A, \mathrm{j} \neq \mathrm{i}\right\}$ using the knowledge $A$ then $A_{i}$ is derivable and those tuples (or data values) are called the derived set which is denoted by $\lambda^{\beta}\left(A_{i}\right)$ where $\beta$ is the time at which the derivation is performed. 

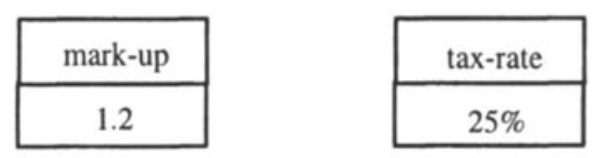

Ipart/sale-price, part/cost-price, mark-up]

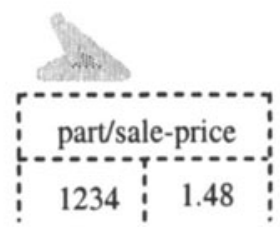

Ipart/sale-price, part/tax-payable, tax-rate]

\begin{tabular}{|c|c|}
\hline \multicolumn{2}{|c|}{ part/cost-price } \\
\hline 1234 & 1.23 \\
\hline
\end{tabular}

Fig. 1. Real and virtual items

\begin{tabular}{|c|}
$\lambda^{\alpha}($ part $)$ \\
\hline part \\
\hline part- \\
number \\
\hline 1234 \\
2345 \\
2468 \\
3456 \\
3579 \\
4567 \\
\hline
\end{tabular}

\begin{tabular}{|c|}
$\Lambda^{\tau}($ part $)$ \\
\hline part \\
\hline part- \\
number \\
\hline 1234 \\
2345 \\
2456 \\
2468 \\
3456 \\
3579 \\
4680 \\
\hline
\end{tabular}

\begin{tabular}{|c|c|}
\multicolumn{2}{c}{$\lambda^{\alpha}$ (part/cost-price $)$} \\
\hline \multicolumn{2}{|c|}{ part/cost-price } \\
\hline $\begin{array}{c}\text { part- } \\
\text { number }\end{array}$ & $\begin{array}{c}\text { dollar- } \\
\text { amount }\end{array}$ \\
\hline 1234 & 1.20 \\
2345 & 2.40 \\
2468 & 3.60 \\
3456 & 4.80 \\
3579 & 5.10 \\
4567 & 6.30 \\
\hline
\end{tabular}

\begin{tabular}{|c|c|}
\multicolumn{1}{c}{$\Lambda^{\tau}$ (part/cost-price $)$} \\
\hline \multicolumn{2}{|c|}{ part/cost-price } \\
\hline part- & dollar- \\
number & amount \\
\hline 1234 & 1.20 \\
2345 & 2.60 \\
2456 & 0.60 \\
2468 & 3.80 \\
3456 & 4.80 \\
3579 & 5.20 \\
4680 & 0.80 \\
\hline
\end{tabular}

Fig. 2. The implementation and the true set

This definition is recursive. In Fig. 1 only part/cost-price, mark-up and tax-rate are stored. This example shows how the decay of the virtual item part/tax-payable is determined by the decay of those three real items and by the decay of those two knowledge items. So if any of those three real items or either of those two knowledge items has decayed in some way then that calculation may yield an incorrect result.

For a knowledge base, its implementation may have decayed because updates that should have been performed were not, or modifications that should not have been performed were. The corruption of a knowledge base by such modifications is not considered here. The failure to perform updates is considered. So updates are changes that should have been performed on the implementation of real items or knowledge items. In addition, incorrect values may be attributed to a knowledge base implementation because the derived tuples for some virtual items were calculated prior to required updates being performed [8]. At time $\alpha$ the true set for a-real or virtualitem $A$ is the set of tuples that should be associated with $A$ at time $\alpha$; it is denoted by $\Lambda^{\alpha}(A)$. In other words, the implementation is what $i$ either stored or derived in the knowledge base, the true set is what should be either stored or derived.

Suppose that the implementation of the real data item part was stored at time $\alpha$. Then at a subsequent time $\tau$ the implementation and the true set may 
be as shown on the left side of Fig. 2. In that Figure the implementation for the data item part contains the part number " 4567 " that should not be there, and does not contain two part numbers which should be there. Suppose that the implementation for the real information item part/cost-price was stored at time $\alpha$. Then at a subsequent time $\tau$ the implementation and the true set may be as shown on the right side of Fig. 2. Likewise the implementation and the true set of a knowledge item may contain tuples that should not be there, and may not contain tuples that should be there.

\section{KNOWLEDGE DECAY}

At time $\tau, \Lambda^{\tau}(A)$ and $\lambda^{\alpha}(A), \alpha \leq \tau$, may not be the same. The implementation of a real item is "correct" as long as its tuples have been correctly stored and maintained [9]. The derived set of a virtual item is "correct" if the knowledge used to derive the tuples for that item has been correctly maintained and the stored data used by that knowledge has been correctly maintained. In reality we may hope that the implementation is correct, and expect that it is incorrect. To measure the extent that the implementation or the derived set are the same as the true set, let $\mathrm{p}_{X / Y}$ be the proportion of those elements in set $\mathrm{X}$ that are also in set $\mathrm{Y}$. Then the difference measure:

$$
\Delta(\mathrm{A}, \mathrm{B})=\sqrt{\mathrm{p}_{\mathrm{A} / \mathrm{B}} \times \mathrm{p}_{\mathrm{B} / \mathrm{A}}}
$$

is unity if both sets are identical and is zero if one set contains no elements from the other; the square root ensures that this measure retains linearity with measured proportion. For example, if each set contains exactly half of the members of the other set then the value of this measure is 0.5 . The value of the difference measure is not necessarily equal to the proportion of valid members in either set because the difference measure takes account of those elements that are not in each set but should be there. The decay of a real or virtual item $A$ at time $\tau$ is:

$\delta_{A}(\tau)=\Delta\left(\lambda^{\tau}(A), \Lambda^{\alpha}(A)\right)$

where $\tau \geq \alpha$ and the difference measure $\Delta$ is as defined above. $0 \leq \delta_{A}(\tau) \leq 1$. If $\delta_{A}(\tau)=1$ then item $A$ is valid. If $\delta_{A}(\tau)=0$ then the set of tuples associated with item $A$ contains no tuples that it should contain and $A$ has decayed completely.

If we know precisely what knowledge decay has occurred then we can usually rectify it [6]. In practice we tend to have some loose expectation $\varepsilon$ of the decay $\delta$. For example, suppose the knowledge item [part/saleprice, part/cost-price, mark-up] is built by applying the knowledge object mark-up-rule to the three items part/sale-price, part/cost-price and mark-up. 
That knowledge item may be used to derive tuples for the information item part/sale-price. We may expect that "within a year the whole part/cost-price relation will be out of date". So our expectation for the decay of the part/cost-price item may be represented by a function with a linear decay of one year's extent. Also, we may expect that "as the 'types' of parts are redesignated, the contents of the part/type relation will decay decreasingly over time so that in a year roughly half of the relation will be out of date and in a 'very long time' the whole relation will be out of date". So our expectation for the decay of the part/type item is represented by a function with an exponential decay with a half-life of one year. The decay estimates for these two examples are:

$\varepsilon_{\text {part }} / \operatorname{cost}-$ price $(\tau)=\left\{\begin{array}{cc}1-\tau & \text { if } 0 \leq \tau \leq 1 \\ 0 & \text { otherwise }\end{array}\right.$

$E_{\text {part } / \text { type }}(\tau)=2^{-\tau}$ for $\quad \tau \geq 0$

If an object has decayed then such decay will contribute to the decay of any item generated by that object. But objects do not have a value set or an implementation. The decay of the item $A$, generated using object $B$, $A=B(C, D, E, F)$ may be analysed completely in terms of the decay of items $C, D, E$ and $F$ and the decay of object $B$. In other words, if items $C, D, E$ and $F$ are valid then the decay of item $A$ will be attributable entirely to the decay of object $\boldsymbol{B}$. So the decay of an object is the decay of any item generated by that object when applied to a set of valid items.

\section{PROPAGATING DECAY ESTIMATES}

It would be convenient if:

$\varepsilon_{o b}(c c, d d)(\tau)=\varepsilon_{o b}(\tau) \times \varepsilon_{c c}(\tau) \times \varepsilon_{d d}(\tau)$

but this product rule is not valid in general because the decay of the components-in the above example the components are $c c$ and $d d-$ may be logically dependent. This product rule is valid if the decay of the object $o b$ and the decay of the two component items are all independent. The decay of $X$ is independent of the decay of $Y$ if $\left(\varepsilon_{X}(\tau) \mid \varepsilon_{Y}(\tau)\right)=\varepsilon_{X}(\tau)$. The decay of $X$ is determined by the decay of $Y$ if $\left(\varepsilon_{X}(\tau) \mid \varepsilon_{Y}(\tau)\right)=\varepsilon_{Y}(\tau)$.

Suppose that object $a b$ is applied to a set of $\mathrm{n}$ component items $\mathrm{C}=\{c c, \mathrm{D}\}$ where $c c$ is a component item and $\mathrm{D}$ is a set of $\mathrm{n}-1$ component items. The general rule for propagating decay estimates through an object operator is:

$$
\begin{aligned}
\mid=\varepsilon_{o b}(C)(\tau)=\varepsilon_{o b}(\tau) \times( & \left.\varepsilon_{C}(\tau) \mid \varepsilon_{o b}(\tau)\right) \\
& =\varepsilon_{C}(\tau) \times\left(\varepsilon_{o b}(\tau) \mid \varepsilon_{C}(\tau)\right)
\end{aligned}
$$


where $\left(\varepsilon_{C}(\tau) \mid \varepsilon_{o b}(\tau)\right)$ is "an estimate of the decay of the set of $\mathrm{n}$ component items $\mathrm{C}$ at time $\tau$ given that the estimate of the decay of object $o b$ at time $\tau$ is $\varepsilon_{o b}(\tau)$." If the knowledge base has been normalised then $\left(\varepsilon_{C}(\tau) \mid \varepsilon_{o b}(\tau)\right)=$ $\varepsilon_{\mathrm{C}}(\tau)$. To propagate decay estimates across a set of component items, suppose that the set $\mathrm{C}$ is a set of $\mathrm{n}$ component items as above. The general recursive rule for propagating decay estimates over such a set is:

$\mid=\varepsilon_{\{c c, D}(\tau)=\varepsilon_{c c}(\tau) \times\left(\varepsilon_{\mathrm{D}}(\tau) \mid \varepsilon_{c c}(\tau)\right)$

where $\left(\varepsilon_{D}(\tau) \mid \varepsilon_{c c}(\tau)\right)$ is "an estimate of the decay of the set of n-1 component items $D$ at time $\tau$ given that the estimate of the decay of the component item $c c$ at time $\tau$ is $\varepsilon_{c c}(\tau)$." In general $\left(\varepsilon_{D}(\tau) \mid \varepsilon_{c c}(\tau)\right) \neq \varepsilon_{D}(\tau)$ even if the knowledge base has been normalised. Now suppose that item $c c$ is virtual and that it is derivable from the set of items $D$ using knowledge item $o b(c c, \mathrm{D})$, ie that $\mathrm{Can}(c c, \mathrm{D})=1$ in $o b(c c, \mathrm{D})$. Then the decay of item $c c$ depends on the decay of object $o b$ and on the decay of the items in the set D. These decay estimates are propagated by:

$\mid=\varepsilon_{c c}(\tau)=\varepsilon_{o b}(\tau) \times\left(\varepsilon_{D}(\tau) \mid \varepsilon_{o b}(\tau)\right)$

If the knowledge base has been normalised then $\left(\varepsilon_{D}(\tau) \mid \varepsilon_{o b}(\tau)\right)=\varepsilon_{D}(\tau)$.

The three rules above for propagating decay estimates follow from the definition of item decay. These three rules lead to complex expressions for decay estimates due to the quantity of conditional expressions-ie expressions involving "|". But the quantity of these conditional expressions may be reduced.

If the knowledge base has been decomposed [10] and if the sub-item relationships have been reduced to sub-type relationships between data items then the object operators and the basis items in the conceptual model are independent with the possible exception of sub-item relationships between data items [5]. So if the knowledge base has been decomposed and there are no sub-item relationships between data items then all the conditional expressions may be removed [1]. For example, if the knowledge base has been decomposed and there are no sub-item relationships between data items then the decay estimate for the virtual information item part/tax-payable is:

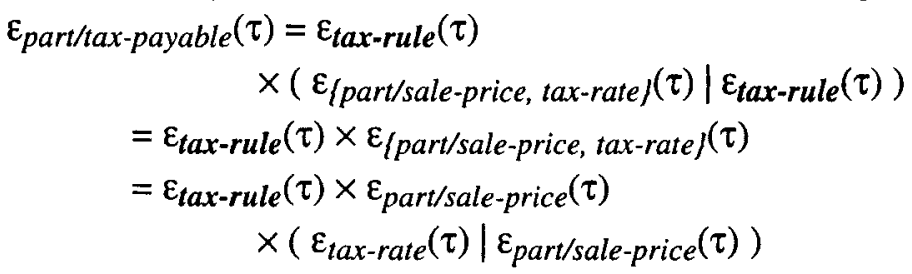




$$
\begin{aligned}
= & \varepsilon_{\text {tax-rule }}(\tau) \times \varepsilon_{\text {part } / \text { sale-price }}(\tau) \times \varepsilon_{\text {tax-rate }}(\tau) \\
= & \varepsilon_{\text {tax-rule }}(\tau) \times \varepsilon_{\text {mark-up-rule }}(\tau) \\
& \times\left(\varepsilon_{\text {/part/cost-price, mark-up } /}(\tau) \mid \varepsilon_{\text {mark-up-rule }}(\tau)\right) \\
& \times \varepsilon_{\text {tax-rate }}(\tau) \\
= & \varepsilon_{\text {tax-rule }}(\tau) \times \varepsilon_{\text {mark-up-rule }}(\tau) \\
& \times \varepsilon_{\text {part } / \text { cost-price }}(\tau) \times \varepsilon_{\text {mark-up }}(\tau) \times \varepsilon_{\text {tax-rate }}(\tau)
\end{aligned}
$$

If sub-item relationships are present or if the knowledge base has not been normalised then the calculations become more involved. For example, suppose the supervisor data item is a sub-item of the person data item. Consider the real person/supervisor item; the implementation of which is populated with 2-tuples (person-id, person-id) where the second person is the "supervisor" of the first. Suppose that this item is built by applying the super information object to the data items person and supervisor:

person/supervisor $=$ super $($ person, supervisor )

then the decay estimate of the person/supervisor information item will be:

$\varepsilon_{\text {super(person, supervisor) }}(\tau)$

$$
\begin{aligned}
& =\varepsilon_{\{\text {person, supervisor }\}}(\tau) \times\left(\varepsilon_{\text {super }}(\tau) \mid \varepsilon_{\{\text {person, supervisor }\}}(\tau)\right) \\
& =\varepsilon_{\text {person }}(\tau) \times\left(\varepsilon_{\text {supervisor }}(\tau) \mid \varepsilon_{\text {person }}(\tau)\right) \times \varepsilon_{\text {super }}(\tau) \\
& =\varepsilon_{\text {person }}(\tau)^{2} \times \varepsilon_{\text {super }}(\tau)
\end{aligned}
$$

where $\left(\varepsilon_{\text {super }}(\tau) \mid \varepsilon_{\{\text {person, supervisor\} }}(\tau)\right)=\varepsilon_{\text {super }}(\tau)$ assuming that the knowledge base has been normalised and the decay of the super operator is independent of the decay of the items to which it is applied; and where ( $\left.\varepsilon_{\text {supervisor }}(\tau) \mid \varepsilon_{\text {person }}(\tau)\right)=\varepsilon_{\text {person }}(\tau)$ because the decay of supervisor is assumed, quite reasonably, to be determined by the decay of person.

\section{CONCLUSION}

Decay is the degradation of integrity over time [11]. In a unified knowledge representation, data, information and knowledge are all represented in a single formalism. Two measures of knowledge integrity are included in a unified knowledge representation. A join operator is defined that enables items in the unified representation to be joined together to form other items. Item join preserves the validity of the two measures of integrity. Decomposition is defined in terms of join. Knowledge base normalisation is defined in terms of decomposition. Normalisation removes hidden relationships from the conceptual model. Hidden relationships can present a maintenance hazard. The representation of a knowledge base using the unified representation, and the normalisation of that representation simplifies the estimation of knowledge decay. 


\section{REFERENCES}

1. Debenham, J.K. "Knowledge Engineering”, Springer-Verlag, 1998.

2. Debenham, J.K. "Knowledge Simplification", in proceedings 9th International Symposium on Methodologies for Intelligent Systems ISMIS'96, Zakopane, Poland, June 1996.

3. Date, C.J., "An Introduction to Database Systems" (4th edition) Addison-Wesley, 1986.

4. Debenham, J.K. "Knowledge Object Decomposition", in proceedings 12th International FLAIRS Conference, Florida, May 1999, pp203-207.

5. Debenham, J.K. "Understanding Expert Systems Maintenance", in proceedings Sixth International Conference on Database and Expert Systems Applications DEXA'95, London, September 1995.

6. Barr, V. "Applying Reliability Engineering to Expert Systems" in proceedings 12th International FLAIRS Conference, Florida, May 1999, pp494-498.

7. Mayol, E. and Teniente, E. "Addressing Efficiency Issues During the Process of Integrity Maintenance", in proceedings Tenth International Conference on Database and Expert Systems Applications DEXA'99, Florence, September 1999, pp270-281.

8. Katsuno, H. and Mendelzon, A.O., "On the Difference between Updating a Knowledge Base and Revising It", in proceedings Second International Conference on Principles of Knowledge Representation and Reasoning, KR'91, Morgan Kaufmann, 1991.

9. Debenham, J.K. "From Conceptual Model to Internal Model", in proceedings Tenth International Symposium on Methodologies for Intelligent Systems ISMIS'97, Charlotte, October 1997, pp227-236.

10. Debenham, J.K. "Representing Knowledge Normalisation", in proceedings Tenth International Conference on Software Engineering and Knowledge Engineering SEKE'98, San Francisco, US, June 1998 pp132-135.

11. Tayar, N. "A Model for Developing Large Shared Knowledge Bases" in proceedings Second International Conference on Information and Knowledge Management, Washington, November 1993, pp717-719. 